\title{
Characterisation of brandies and wood extracts by capillary electrophoresis*
}

\author{
M. Rosário Bronze ${ }^{1,2}$ and L.F. Vilas Boas ${ }^{1,3}$ \\ ${ }^{1}$ Instituto de Biologia Experimental e Tecnológica, Apartado 12, 2780 Oeiras, Portugal \\ ${ }^{2}$ Faculdade de Farmácia de Lisboa, Av. das Forças Armadas, 1600 Lisboa, Portugal \\ ${ }^{3}$ Instituto Superior Técnico, Av. Rovisco Pais, 1095 Lisboa, Portugal
}

\begin{abstract}
Wood extracts and brandies aged in barrels from oak and chestnut wood toasted at three different levels were analysed by Capillary Electrophoresis and UV absorption. The results were discussed taking into account the composition of phenolic compounds extracted from wood: hydrolysable tannins, aldehydes and acids (benzoic and cinnamic). The relative importance of measured variables is discussed in terms of ability to differentiate various samples (origin of the wood and the toasting level used in the production of barrels) as well as making predictions about sensorial characteristics of brandies.
\end{abstract}

Key words. capillary electrophoresis - principal components analysis - polyphenols in wood and brandy.

\section{Introduction}

The content of phenolic compounds in natural products may be estimated by measurement of UV absorption $(280 \mathrm{~nm})$ or measured by Folin-Ciocalteau method. In order to analyse each compound, separation techniques as High Performance Liquid Chromatography (HPLC) [1-5,11,14,17,20-23,25-28] or Capillary Electrophoresis [6-10] have been used.

Recently we reported [11] that Capillary Electrophoresis could be used in the analysis of polyphenols in brandies and wood extracts. In this paper, we extend this study to the analysis of brandies and wood extracts by UV absorption and Capillary Electrophoresis in order to show the effect of different woods at three toasting levels on the composition of brandies or extracts.

The quality of brandies depends on factors that will have to be considered when results from chemical analysis are discussed: those factors are related with the characteristics of wines used to produce the distillates, the whole process of distillation, and finally the ageing process.

Traditionally the ageing process of brandies is carried out in wood barrels made of oak (e.g. Quercus robur, Quercus petreae, Quercus alba) and sometimes chestnut (Castanea sativa). During ageing, the distillate containing about $75 \%$ ethanol slowly extracts components from the wood used to make the barrel: a large variety of phenolic compounds (aldehydes, benzoic acids, cinnamic acids, and hydrolysable tannins) may be found in such solutions and are very important as they may contribute to sensorial characteristics of brandies. Molecules as lignin and hemicelluloses are also depolymerised and extracted.

These compounds extracted from wood may react in solution and therefore chemical composition may change. Finally, oxidation reactions occur as small amounts of oxygen pass through the wood barrel walls $[12,13]$.
The influence of chemical composition of wood on sensorial properties of wines [14-16], whiskies [17], brandies [18] and cognacs [19], has been widely studied and correlated with quality appreciation by the consumers.

However, the characteristics of wood depend on many variables: species of trees [20-22,24], their geographical origin $[21,23,24]$ (kind of soil, length of growing season, temperature, average rainfall), their age $[13,24]$, process used in seasoning of wood and cutting techniques [12,14,24], wood toasting level [12,19,24-26], how often barrels have been used and for what kind of drink [27,28].

Wood barrel dimensions are important due to the influence on the area of contact wood/solution and the amount of oxygen carried into solution [17].

The industry of cooperage is very important to the producers of wine and distilled drinks but factories are usually small and use traditional techniques not easy to standardise.

Flavour, colour and taste of brandies are strongly influenced by the toasting level of wood, so the producers may choose which is the toasting level more adequate to obtain a product according to the preferences of consumers.

When wood is toasted, its temperature may rise at about $200{ }^{\circ} \mathrm{C}$ or higher, destruction of wood begins at about $250{ }^{\circ} \mathrm{C}$ and the changes in chemical composition of wood are strongly influenced by temperature. Light toast means that fire is only used for about $5 \mathrm{~min}$, and at the end, the colour of the wood is not different from the initial. In medium toast, fire is used for about $15 \mathrm{~min}$ and produces woods with darker colour. Times longer than 25 min produce much darker woods and these barrels submitted to strong toasting are often preferred for the production of brandies.

Processes used to treat the wood are traditional and based on personal experience: the time used to treat the wood with fire is often controlled by the smell produced by the wood 
while toasting and depends strongly on workers experience. During this process some degradation compounds produced from polyosides, lignin, and lipids have characteristic flavours: toast almond (furanic aldehydes), cacao, praline, fresh bread, coffee, toast and caramel are associated to degradation products of sugars, and vanillin is a degradation product of lignin [1]. Therefore all this process is very irreproducible in a particular industry, and on comparison of different industries greater differences may certainly be found.

Brandies aged in different barrels made of similar wood and treated at the same toasting level may have different sensorial characteristics. This would be inconvenient if noticed by consumers: therefore blending, controlled by sensorial analysis is required in order to have a product maintaining the same quality.

This work is part of a study of brandies from an Appellation Controlled Region in Portugal (Lourinhã) where the acidic wines produced were considered very adequate for the production of brandies of good quality.

\section{Experimental}

\section{Chemicals}

All reagents were reagent grade. Derivatives from cinnamic acid, benzoic acid and aldehydes were obtained from Sigma Chemical (St. Louis, USA) or from Aldrich-Chemie (Steinheim, Germany). Other standards as acetone p.a. from Merck (Darmstadt, Germany), naphtol (Analar, BDH, England) and ellagic acid from Aldrich-Chemie (Steinheim, Germany), were used.

Standard solutions were prepared by dissolving compounds in methanol p.a. grade from Riedel-de-Haën (Seelze, Germany).

For the identification of castalagin and vescalagin, as they are not marketed, separation of an aqueous oak wood extract was performed on a Sephadex LH-20 (Pharmacia LKB, Uppsala, Sweden) column. Fractions were collected and analysed by HPLC [3] and EC. Compounds were identified comparing results from chromatograms and electropherograms.

Running electrolyte solutions were prepared from sodium tetraborate decahydrate p.a. grade (Aldrich-Chemie, Steinheim, Germany) at a concentration of $10 \mathrm{mM} \mathrm{pH}=9.2$ with 5\% ethanol (Riedel-de-Haën, Seelze, Germany).

Water used in all experiments was purified with a MilliQ apparatus (Millipore, Bedford, USA) with a conductivity $18 \mathrm{M} \Omega$.

\section{Preparation of wood samples and collection of wood samples}

At a cooperage industry (J.M.A. Gonçalves) 63 wood barrels $(250 \mathrm{~L})$ were made of wood from different trees, toasted at 3 levels (light, medium and strong) and three replicates were obtained for each wood and toasting level.

Before and after toasting process of barrels, shaves were cut in order to get samples of wood that were kept in plastic bags until preparation for analysis.
Woods used in this work were French oaks (from Limousin and Allier), American oak, and Portuguese oaks from three different regions and currently being studied in order to identify their botanical species. For the sake of comparison chestnut was also used for making barrels, and were treated in the same way as oak woods.

Before analysis, shaves were reduced to powder. Extracts of wood were prepared using a model solvent system consisting of $55 \%$ ethanol (v/v) acidified until $\mathrm{pH}=4.2$ as described previously [11]. $5 \mathrm{~g}$ of powdered sample were extracted with $10 \mathrm{ml}$ of the model extracting solution for 3 hours at room temperature. Extracts were filtered through Millipore $0.45 \mu \mathrm{m}$ membranes and were analysed. If necessary extracts were diluted with the model extracting solution.

\section{Preparation of brandies and sampling}

Wines from the region of Lourinhã were distilled and used to fill the barrels previously prepared. Ethanolic content of the distillate was $75 \%(\mathrm{v} / \mathrm{v})$. After one year ageing in the same cellar, 63 samples of brandies were collected, filtered $(0.45 \mu \mathrm{m}$ membrane from Millipore) and analysed. If necessary, brandies were diluted with ethanolic solution $75 \%$ $(\mathrm{v} / \mathrm{v})$.

\section{Equipment}

- Capillary electrophoresis equipment PRINCE from Lauerlabs (Emmen, Netherlands) with an UV detector operating at fixed wavelength and an uncoated fused-silica capillary (total length $=100 \mathrm{~cm}$, effective length $=85 \mathrm{~cm}$, $75 \mu \mathrm{m}$ I.D., $340 \mu \mathrm{m}$ O.D.) were used. Electropherograms were recorded with an integrator CR4A-Chromatopac from Shimadzu (Kyoto, Japan).

- Spectrophotometer Beckman DU-70 from Beckman Instruments, Inc., (Fullerton, USA) and quartz cells of 1.0 and $0.1 \mathrm{~cm}$ were used.

\section{Conditions of analysis by capillary electrophoresis}

Standard solutions and samples were introduced at the anode extremity of the capillary by applying a $10 \mathrm{mBar}$ differential pressure for 12 seconds. Separations were carried out at $35^{\circ} \mathrm{C}$, by applying a voltage of $25 \mathrm{kV}$. Detection was performed at $254 \mathrm{~nm}$ using a detection window in the cathode extremity of the capillary. Running electrolyte solutions consisted of sodium tetraborate decahydrate $10 \mathrm{mM} \mathrm{pH}=$ 9.2 with $5 \%$ of ethanol (organic modifier). In these conditions of analysis current intensity was about $19.4 \mu \mathrm{A}$.

Capillary conditioning was made as mentioned previously [11], and its performance was controlled at the beginning of every working day with a standard mixture solution consisting of $20 \mathrm{ppm}$ from each standard: naphtol, sinapinaldehyde, coniferaldehyde, syringaldehyde, vanillin, sinapic acid, syringic acid, vanillic acid, p-hydroxybenzoic acid, gallic acid. To monitor electroosmotic flow, acetone was used.

Some compounds separated by capillary electrophoresis were identified spiking sample with standards. 


\section{Measurement of absorbance at different wavelengths}

Absorbance of wood extracts and brandies were measured $(254,280,360,440 \mathrm{~nm})$ and spectra ranging from 220-500 nm were traced against extracting solution. Wood extracts if necessary were diluted with extracting solution.

\section{Statistical treatment of the results}

As there was a great number of results and samples, Principal Component Analysis (PCA) a statistical treatment of results was used (Rohlf F, "NTSYS-pc. Numerical Taxonomy and Multivariate Analysis System, version 1.7", Exeter Software, NY, 1992).

\section{Results and discussion}

The effect of toasting level on the colours of brandies or wood extracts may be observed visually and the spectra of different wood extracts were compared by measuring the absorbances at $280 \mathrm{~nm}$. Analysis of phenolic compounds is very difficult due to the great variety of these molecules existing in plants and also because of the reactivity of these compounds. The spectra of different wood extracts solutions were used for a first comparison and although the measurement of absorbance at $280 \mathrm{~nm}$ is used to quantify phenolic content of samples, detection in capillary electrophoresis was monitored at $254 \mathrm{~nm}$ as some compounds (e.g. ellagitannins, aldehydes) presented higher absorptions at this wavelength.

By comparing the absorbances measured at $280 \mathrm{~nm}$ it was concluded that the concentration of wood extracts was in the order:

Portuguese oak $>$ French oak, Limousin $>$ French oak, Allier $>$ American oak and these observations are consistent with earlier work [23]. Major differences are also observed for different toasting levels of oak wood as absorbance at $280 \mathrm{~nm}$ increases.

Spectra of chestnut wood extracts and oak were compared. Although absorbance at $254 \mathrm{~nm}$ may be similar for both kinds of woods, absorbance for chestnut at $280 \mathrm{~nm}$ was always higher. If the ratio of absorbances $254 \mathrm{~nm} / 280 \mathrm{~nm}$ was considered their range was about $1.5-1.6$ for American oak extract and $1.2-1.3$ for chestnut. When electropherograms at $254 \mathrm{~nm}$ and $280 \mathrm{~nm}$ from these samples were compared, chestnut extracts presented higher concentrations of compounds absorbing at $280 \mathrm{~nm}$.

Figure 1 illustrates the electropherograms obtained by analysis of two samples of wood extracts showing that different groups of compounds correspond in the electropherogram to different ranges of migration times: A. compounds that are not separated in these conditions of analysis and, as they are carried by electroosmotic flow as neutral molecules; B. alcohols; C. benzoic and cinnamic aldehydes; D. benzoic and cinnamic acids; E. an apparent "large peak" including a large number of compounds that may have a complex structure and are incompletely separated by this technique. Some important compounds as ellagic acid, gallic acid and ellagitannins (castalagin and vescalagin) are important peaks with similar migration times.
As mentioned in previous work [11] compounds with free $\mathrm{OH}$ groups may form borate complexes with running electrolyte: these complexes have higher negative charges than the original compounds. Differences in ionic mobilities and migration times can be discussed taking into consideration the balance between the increase of negative charge and size of the ion.

The effect of toasting on chemical composition of wood is also shown in figure 1, as the same wood was treated to different toasting levels. Electropherograms show differences that may be ascribed to an increase of ellagic acid and aldehydes reported earlier and due to degradation of lignin and ellagitannins [12,19].

It was observed that most of samples treated at high temperatures show an increase in the "large and wide peak" corresponding to a profile that will include a great number of similar compounds. A plausible explanation for this observation is the formation of a large number of molecules with similar chemical behaviour as the result of thermal decomposition of compounds with higher molecular weights, including polymeric molecules. However, for a small number of samples of wood extracts the "large and wide peak" became smaller after wood treatment. A plausible explanation is that the wood may have been toasted for a longer period or at higher temperature, as the toasting process is not easily controlled, and these compounds could be partially destroyed as a result of carbonization process of wood.

The electropherograms of oak extracts showed compounds migrating between $12-14$ min not detected in chestnut extracts. Figure 2 illustrates electropherograms of different oak species and chestnut wood extracts diluted $(1: 10)$ with extracting solution. Extracts from different species of oak have similar chemical components although differing in concentration. Chestnut presented higher relative concentration of gallic acid and vescalagin.

A total of 63 brandies and 126 wood extracts were analysed by UV-absorption measurements and capillary electrophoresis: Principal Component Analysis (PCA) was found very convenient for the comparison of the large number of experimental results obtained from the analysis of these samples as it was possible to discriminate groups of samples and conclude about similarities as well as the relative importance of variables contributing for this discrimination.

Before describing the application of PCA in this study it should be noticed that:

1. Sampling procedure in brandies should be less influenced by variations than wood shavings. Although brandies have not yet completed their ageing time ( 24 months at least), their chemical composition will be more stable than the wood extracts solutions prepared in the laboratory just before analysis.

2. Results obtained from chemical analysis of trees from different species (oak and chestnut) and trees (oak) from different origins will give us an idea on how these variables may cause variations on chemical composition of wood extracts. As different toasting levels were used, it is possible to conclude which variables are more influenced by heating process.

3. Although there are some problems mentioned earlier related with the representative sampling of wood extracts, it 


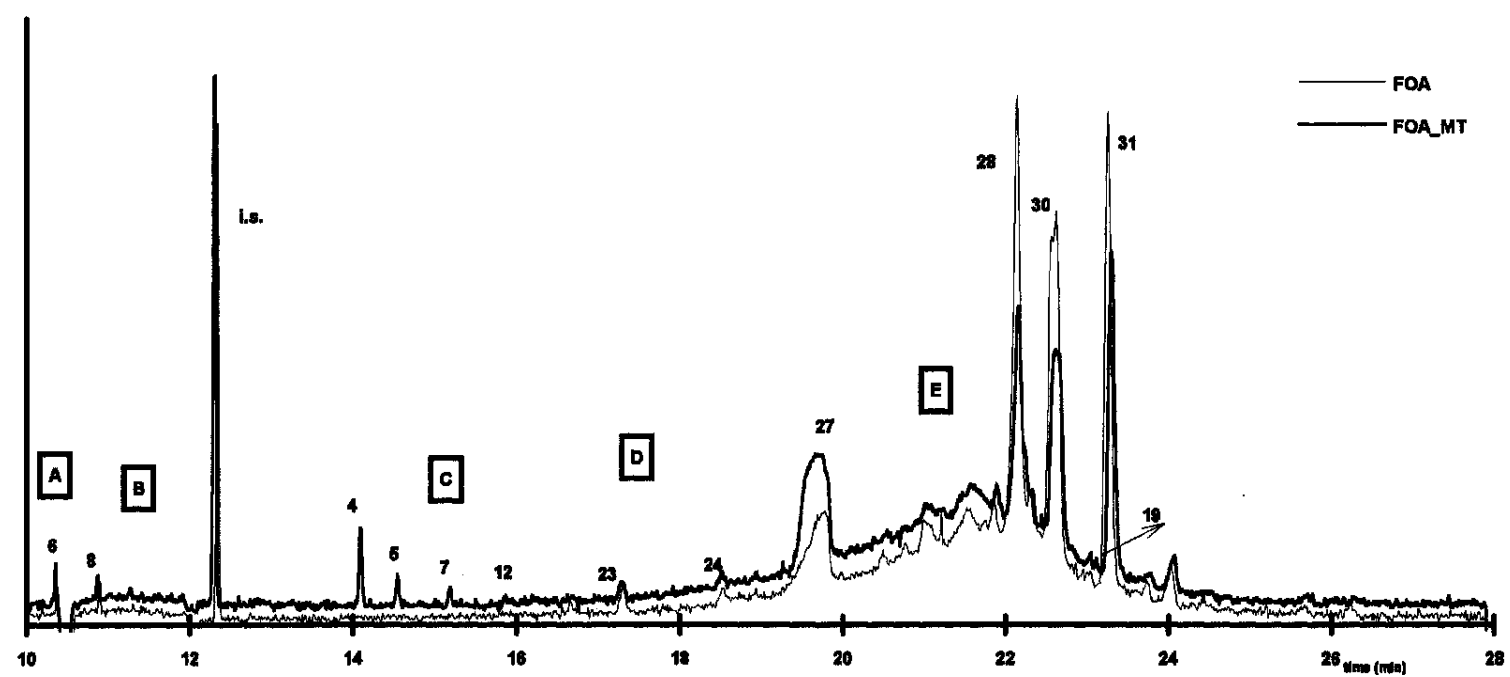

Fig. 1. Electropherograms of an ethanolic extract of French oak from Allier, before (FOA) and after medium toast (FOA_MT). Peak identified as i.s. was naphtol used as internal standard and the others were identified as in table I.

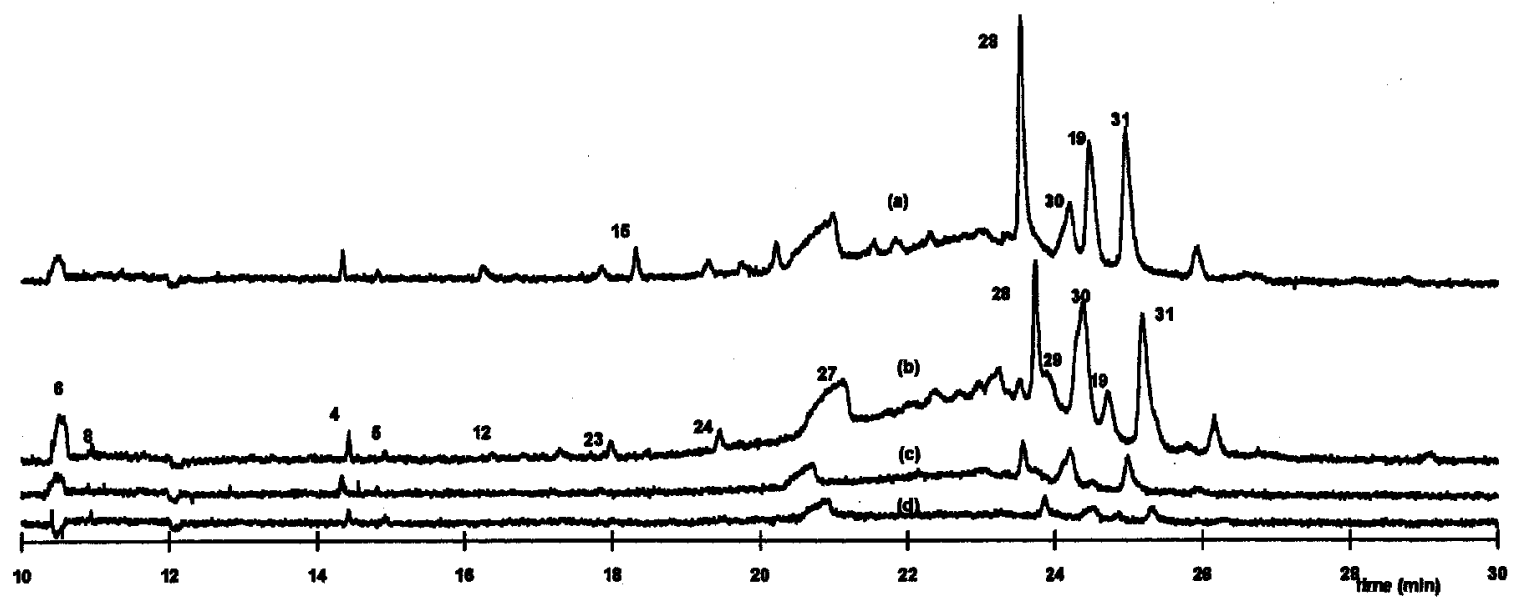

Fig. 2. Electropherograms of ethanolic extracts of wood from chestnut (a), Portuguese oak (b), French oak (c) and American oak (d). All woods were strongly toasted and extracts were diluted before analysis. Compounds identified as in table I.

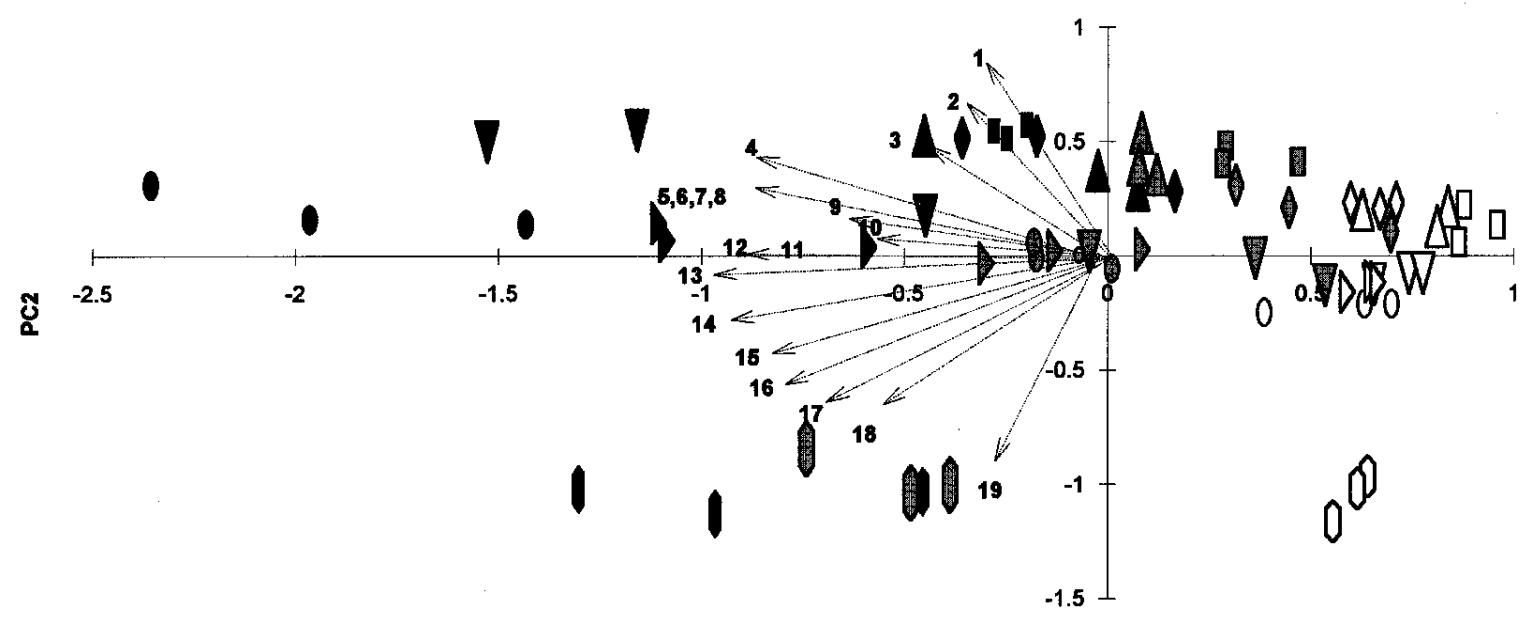

PC1

Fig. 3. Projection of samples of brandies in the plane defined by Principal Component 1 (PC1) and Principal Component 2 (PC2). Different symbols were used to identify the different samples: $\boldsymbol{M}$ Portuguese oak woods, $\boldsymbol{\Lambda}$ French oaks (respectively Limousin and Allier); American oak; chestnut. Different toasting levels of the wood used in the aging process were identified by the background color of the symbol: black for strong toast; gray for medium toast; white for light toast. Eigenvectors corresponding to each variable measured are represented (see table I for identification). 


\section{Original articles}

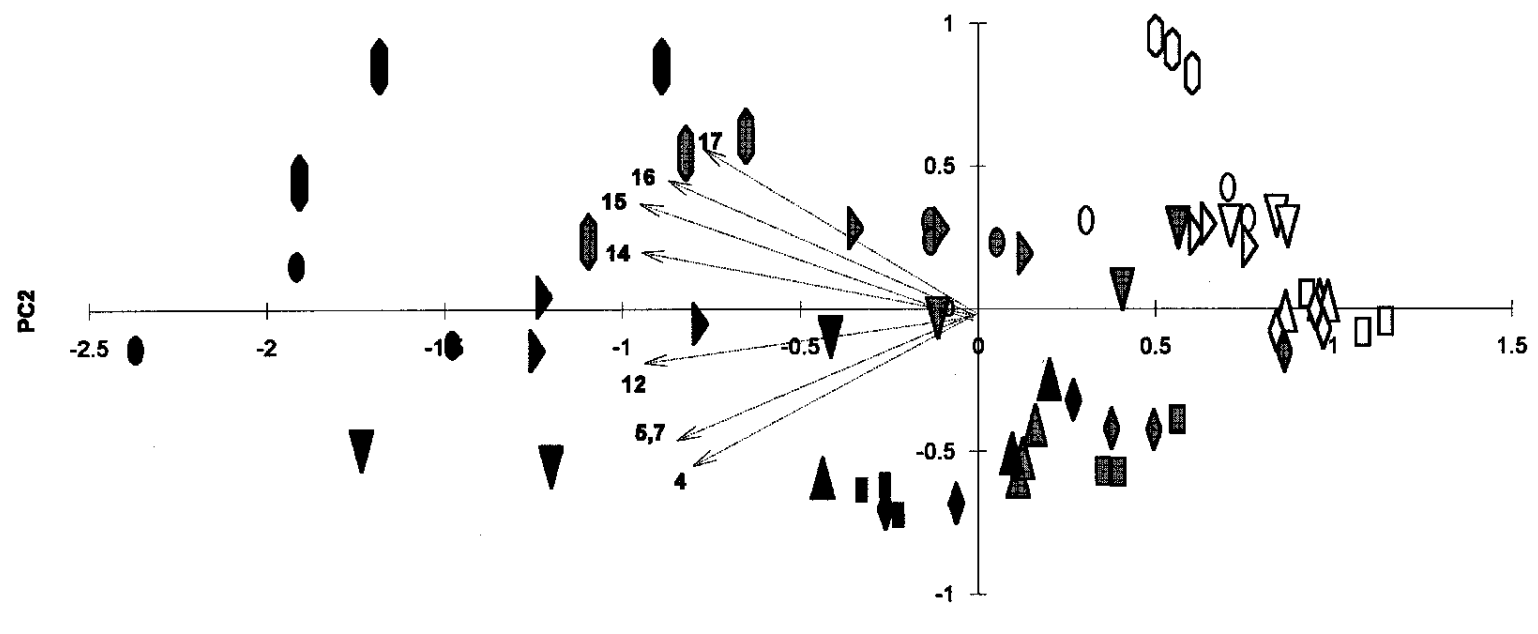

PC1

Fig. 4. Projection of samples of brandies in the plane defined by Principal Component 1 (PC1) and Principal Component 2 (PC2) considering only 8 variables. For identification of samples see legend from figure 3. Variables are identified in table I.

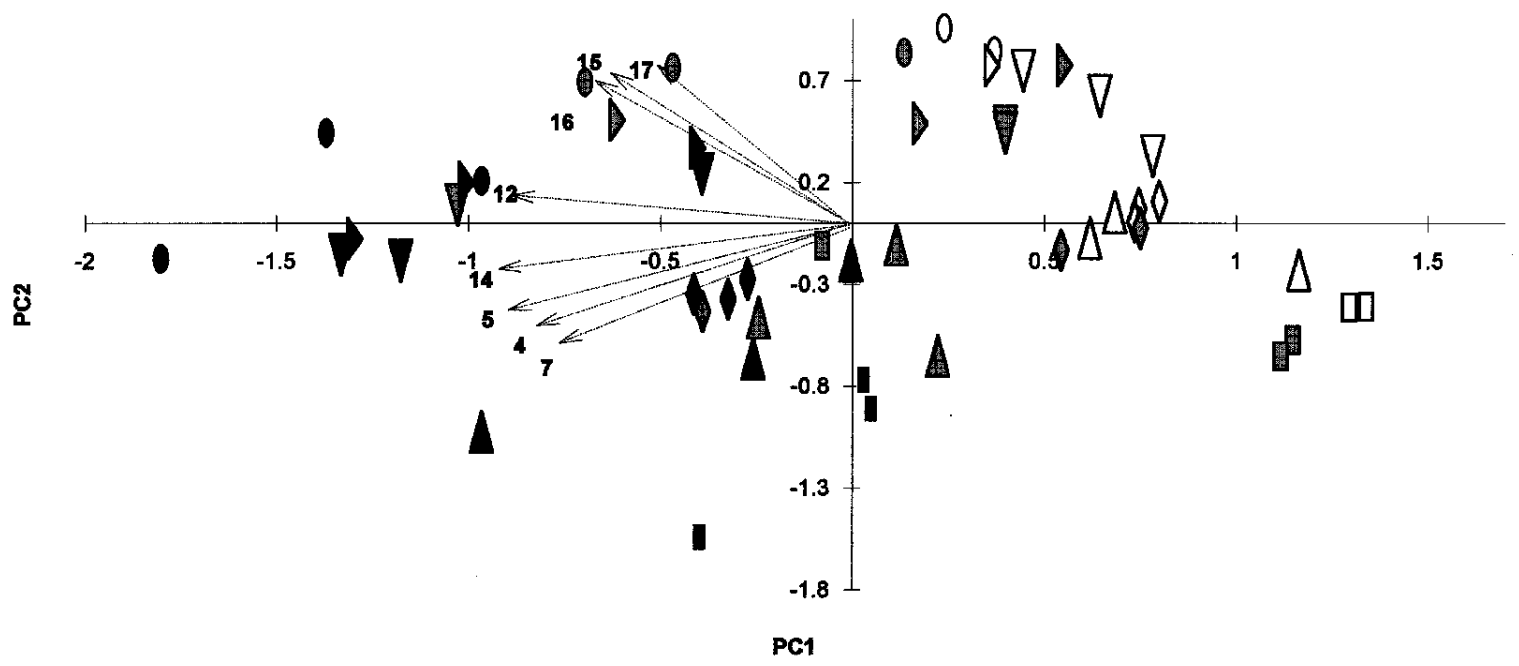

Fig. 5. Projection of samples of wood extracts in the plane defined by Principal Component 1 (PC1) and Principal Component 2 (PC2) considering only 8 variables. For identification of samples see legend from figure 3 . Variables are identified in table I.

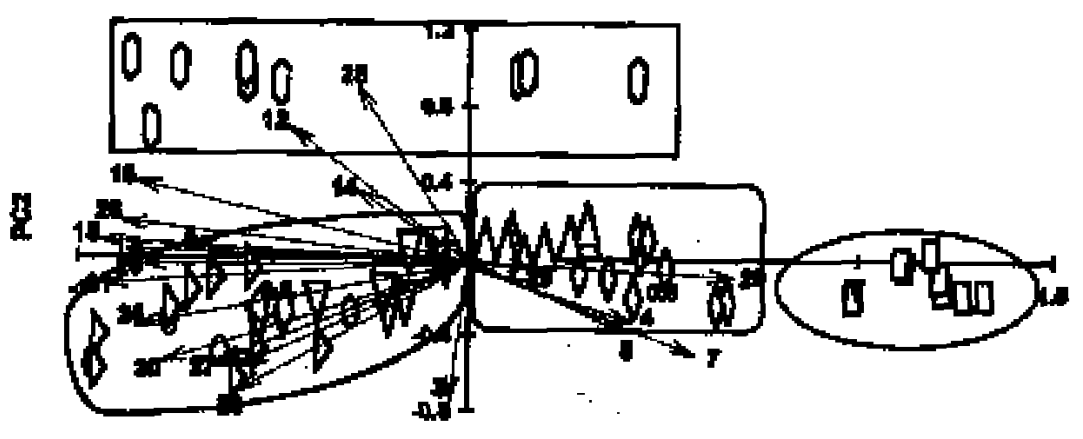

PSI

Fig. 6. Projection of samples of wood extracts not toasted in the plane defined by Principal Component 1 (PC1) and Principal Component 2 (PC2) considering 21 variables. For identification of samples see legend from figure 3. Variables are identified in table I. 


\section{Original articles}

Table I. Identification of variables used in the PCA.

\begin{tabular}{rccc}
\hline$n^{\circ}$ & Identification & $n^{\circ}$ & Identification \\
\hline 1 & ferulic acid & 17 & "large peak" \\
2 & vanillic acid & 18 & peak at 26.0 min $\left(^{*}\right)$ \\
3 & peak at 10.9 min $\left(^{*}\right)$ & 19 & gallic acid \\
4 & coniferaldehyde & 20 & peak at 16.2 min $\left(^{*}\right)$ \\
5 & sinapinaldehyde & 21 & peak at 16.4 min $\left(^{*}\right)$ \\
6 & neutral compounds & 22 & peak at 16.6 min $\left(^{*}\right)$ \\
7 & syringaldehyde & 23 & peak at 17.4 min $\left(^{*}\right)$ \\
8 & peak at 11.3 min $\left(^{*}\right)$ & 24 & peak at 18.1 min $\left(^{*}\right)$ \\
9 & peak at 13.9 min $(*)$ & 25 & peak at 18.5 min $\left(^{*}\right)$ \\
10 & sinapic acid & 26 & peak at 19.5 min $\left(^{*}\right)$ \\
11 & syringic acid & 27 & ellagic acid \\
12 & vanillin & 28 & vescalagin \\
13 & absorbance at $360 \mathrm{~nm}$ & 29 & peak at 22.1 min $\left(^{*}\right)$ \\
14 & absorbance at $440 \mathrm{~nm}$ & 30 & peak at 22.4 min $\left(^{*}\right)$ \\
15 & absorbance at $254 \mathrm{~nm}$ & 31 & castalagin \\
16 & absorbance at $280 \mathrm{~nm}$ & & \\
\hline
\end{tabular}

(*) Although these compounds have not been identified yet, they have been taken as variables for comparison of samples as Capillary Electrophoresis gives reproducible results and is very efficient as separative technique.

is important to consider the results of their analysis as these extracts are prepared in a short time and solutions are much more concentrated than the corresponding brandies.

Application of PCA to the results obtained from the analysis of brandies showed that only the first three principal components could be considered relevant as accounted for $89.4 \%$ of the overall variance.

Figure 3 shows the projection of samples on the plane defined by the Principal Component 1 (PC1), which accounted for an individual variance of $56.2 \%$, and Principal Component 2 (PC2) which accounted for an individual variance of $22.8 \%$. Some conclusions can be pointed out:

1. brandies aged in barrels made with chestnut are easily distinguished from the brandies aged in barrels made with oak wood from different origins;

2. sample distribution along PC1 shows that toasting level is important to discriminate the samples;

3. the origin of samples is important not only in PC1 but also in PC2 direction.

4. there are some difficulties to separate samples in order of the toasting level of the wood used in the ageing process. This can be explained by the characteristics of the industrial toasting process that is not reproducible, even in the same cooperage factory.

If we look carefully to the projection of variables on PC1 and PC2 plane (Fig. 3), it seems important to notice that:

1. contribution of variables 1, 2 and 3 are related, aldehydes (except vanillin) are very close to the peak of neutral compounds, peak of gallic acid is separated from the other variables that include mostly UV absorbance measurements.
2. it was mentioned before that PC1 was related with toasting level and PC2 was related with the origin of wood. From figure 3 it is clear that aldehydes content and UV absorption values are related with toasting level, while the other group of variables separates samples concerning their origin.

Brandies aged in chestnut wood were separated based on their vector loadings for gallic acid, compounds from large peak, peak 18 (compound not identified), absorbances at: $280 \mathrm{~nm}$ (total phenolic compounds content), $254 \mathrm{~nm}$, and $440 \mathrm{~nm}$.

In order to compare the results of brandies and wood extracts, only 8 variables were considered among the 19 used before. Those 8 variables were: UV absorption (at $254 \mathrm{~nm}, 280 \mathrm{~nm}$ and $440 \mathrm{~nm}$ ), the area of the "large peak", and the aldehydes content: sinapaldehyde, coniferaldehyde, vanillin, syringaldehyde. Although gallic acid and peak 18 discriminated chestnut samples they were not considered among those 8 variables as it was not possible to quantify those peaks in all wood extracts.

Considering these 8 variables, projection of samples is shown in figure 4 and the relative positions of points are similar to the projection obtained with 17 variables, although samples of brandy aged in American oak are not so well separated from the French oak samples. It was possible to conclude that those 8 variables seemed to have relevant information concerning chemical composition for the study of the different brandies. The first three principal components could be considered relevant as accounted for $97.2 \%$ of the overall variance, contributing PC1 with $75.6 \%$ and PC2 with $19.32 \%$.

Although brandies aged in Portuguese woods are separated from those aged in French oaks, there is no clear separation between brandies aged in oaks from three different regions of Portugal.

In brandies aged in chestnut wood with a light toasting level, aldehydes content was very low and it was difficult to distinguish peaks from baseline noise. For the same samples, absorption measurement at $280 \mathrm{~nm}$ was higher than the value obtained at $254 \mathrm{~nm}$ while for brandies aged in chestnut submitted to medium and strong toast the opposite was observed.

Brandies aged in barrels made with American oak presented lower concentration of compounds of the "large peak" but proportionally they were concentrated in aldehydes.

It seemed to be important to compare results of chemical composition of brandies and the corresponding wood extracts. There was a gain a great number of results and PCA was applied to discuss the wood extracts composition. The same 8 variables used to compare samples of brandies were initially considered (Fig. 5). Grouping of different variables is more similar to the results obtained with brandy if results from chestnut are not taken into account. In fact, for chestnut the area of the peak identified as vanillin is larger.

In wood extracts, UV absorption at $440 \mathrm{~nm}$ seems to be more related with toasting level than with the origin of wood, while in brandies absorption at this wavelength is more related with wood origin. 
When instead of only 8 variables we use the 24 variables measured for 126 samples (extracts of woods toasted and not toasted), the first three principal components represented $75.7 \%$ of total variance. The projection of results on the plane defined by the PC1 (which accounted for an individual variance of $32.9 \%$ ) and PC2 (which accounted for an individual variance of $27.7 \%$ ) gives a complex figure that will not be presented here. Anyway, it would be interesting to notice that such projections on $\mathrm{PC} 1 / \mathrm{PC} 2$ plane shows:

- the extracts of wood not toasted were separated from the extracts of wood toasted at different levels;

- it was possible to distinguish four groups of samples: Portuguese oak, French oak, American oak, chestnut;

- considering the projections of the variables, it was possible to distinguish five groups of variables with ellagic acid contribution appearing separated from the others.

PC1 discriminates samples considering their toasting level based on ellagic acid, aldehydes and UV absorption at 440 $\mathrm{nm}$. PC2 is more related with the origin of wood and discrimination occurs based on absorption measurements, compounds from the large peak and ellagitannins content. However the two components are not independent from one another as some variables contribute to differentiate types of woods and toasting levels.

There was also a group of variables that seemed not to contribute in an important way to the separation of different samples.

Correlation between aldehydes content and ellagic acid is good for Portuguese and chestnut toasted woods, but there is no apparent correlation for French or American woods.

American oak wood extracts presented a lower peak area of the compounds from the "large peak", although aldehydes content was not so different from the other samples of wood extracts, and the same was observed in the corresponding brandies.

However from results obtained it was difficult to make conclusions about extracts from woods not toasted, and therefore they were analysed separately by PCA in order to obtain figure 6 that is much simpler to interpret. In figure 6 , there are 4 groups of samples (these results include a total variance of $75.2 \%$ for the three principal components).

Characterisation by chemical analysis of food products is very often intended to get clues about sensorial properties or evaluation of quality. As the brandies used in this study will be evaluated by a sensorial analysis panel, we shall discuss briefly some properties that may be anticipated from the chemical composition observed:

- Brandies aged in strongly toasted wood will probably be preferred as their aldehyde content is higher, and from literature it is known that aldehyde content contribute in a positive way to their appreciation (however these preferences may depend on consumer habits).

- If colour is considered an important parameter for brandies quality certainly strong toasts of woods are chosen, and concerning the type of wood, chestnut will be the one preferred. However sometimes compounds responsible for colour may contribute with a certain astringency to the taste, and this is an important parameter to consider when evaluating this kind of samples.

- If preferences of a sensory panel are directed specially to flavour with less concern for colour it can be anticipated that brandies aged in French and American woods will be preferred.

Within the activities of this project corresponding to the study of brandies from Lourinhã region, these same samples will also be analysed by HPLC and gas chromatography. These techniques may provide information for a complete chemical characterisation of samples and, on the other hand, may be very useful in validating information about variables measured by capillary electrophoresis for sample comparison and classification.

\section{Conclusions}

Although the effect of species, geographical origin of the tree and temperature effects in wood composition, have been well known for a long time, this work confirms results presented before [11] and shows Capillary Electrophoresis as an adequate technique for the analysis of brandies or wood extracts.

The results from Capillary Electrophoresis and UV absorption treated by Principal Component Analysis showed there were differences according to different origin of woods as well with toasting level.

Due to the characteristics of the industrial processing of wood sometimes there is not a clear separation between woods with different toasting levels: there is a clear separation between samples corresponding to woods subjected to strong and light toast, but distinction between medium and light or medium and strong is not always clear.

There was a relation between brandies and corresponding wood extracts although extracting conditions differ in each process.

Although some more information is required for a full characterisation of these samples the results obtained by UV absorption and Capillary Electrophoresis give already some detailed information on their phenolic composition.

Wood containers have a great importance for industries producing brandies. There is a need for a technique of evaluation of barrels as a method of predicting the quality of brandies aged in such barrels or giving hints about questions to ask the sensorial analysis panel. Capillary Electrophoresis is likely to be a suitable technique for this purpose.

\section{Acknowledgements}

The authors express their gratitude to Eng. Pedro Belchior for the helpful discussions and Ms. Felismina Gonçalves for the explanations concerning the industrial process of cooperage.

The financial support within PAMAF program (project IED 2052) is gratefully acknowledged. 


\section{References}

1. Scalbert, A.; Monties, B.; Favre, J.-M. Phytochemistry 1988, 27, 3483-3488.

2. Belchior, A. P.; Spranger, M. I.; Carvalho, E. C.; Leandro, M. C. in Cantagrel R. Ed.; Élaboration et Connaissance des Spiritueux, Recherche de la qualité, tradition et innovation; BNIC, diffusion Tec et Doc Lavoisier, 1993; p 479.

3. Scalbert, A.; Duval, L.; Peng, S.; Monties, B.; Penhoat, C. J. Chromatogr. 1990, 502(1), 107-119.

4. Bronze, M. R.; Vilas Boas, L. F. In: Lonvaud-Funel A. Ed.; Oenologie 95, Bordeaux, 1995, Paris, 1996; p 641.

5. Vivas, N.; Glories, Y.; Bourgeois, G.; Vitry, C. J. Sci. Tech. Tonnellerie 1996, 2, 25-49.

6. Seitz, U.; Oefner, P.; Nathakarnkitkool, S.; Bonn, G. K. Electrophoresis 1992, 13, 35-38.

7. Jen, J.-F.; Hsu, Y.-H.; Lee, M.-R. J. Chromatogr. A 1996, 734, 375-380.

8. Liang, H.-R.; Sirén, H.; Riekkola, M.-L.; Vuorela, P.; Vuorela, H.; Hiltunen, R. J. Chromato gr. A 1996, 746, 123-129.

9. Horie, H.; Mukai, T.; Kohata, K. J. Chromatogr. A 1997, 758, 332-335.

10. Masselter, S.; Zemann, A.; Bobleter, O. Chromatographia 1995, 40(1-2), 51-58.

11. Bronze, M. R.; Vilas Boas, L. F.; Belchior, A. P. J. Chromatogr. A 1997, 768, 143-152.

12. Vivas, N.; Glories, Y. Rev. Fr. Oenol. 1993, 142, 33-38.

13. Singleton, V. L. Am. J. Enol. Vitic. 1995, 46(1), 98-115.

14. Puech, J.-L. Am. J. Enol. Vitic. 1987, 38(3), 236-238.
15. Pocock, K. F.; Sefton, M. A.; Williams, P. J. Am. J. Enol. Vitic. 1994, 45(4) 429-434.

16. Chatonnet, P. J. Intern. Sc. de la Vigne et du Vin, Le bois et la qualité des vins et des eaux-de-vie, 1992; pp 81-91.

17. Withers, S. J.; Piggott, J. R.; Conner, J. M.; Paterson, A. J. Inst. Brew. 1995, 101, 359-364.

18. Belchior, A. P.; Puech, J.-L. Ciência Téc. Vitiv. 1983, 2(2), 5765.

19. Puech, J.-L. J. Intern. Sc. de la Vigne et du Vin, Le bois et la qualité des vins et des eaux-de-vie, 1992; pp 39-49.

20. Simon, B. F.; Conde, E.; Cadhia, E.; Garcia-Vallejo, M. C. J. Sci. Tech. Tonnellerie 1995, 2, 1-10.

21. Miller, D. P.; Howell, G. S.; Michaelis, C. S.; Dickmannn, D. I. Am. J. Enol. Vitic. 1992, 43(4), 333-338.

22. Laszlavick, M.; Gál, L.; Misik, S.; Erdei, L. Am. J. Enol. Vitic. 1995, 46(1), 67-74.

23. Marco, J.; Artagona, J.; Larrechi, M. S.; Ruis, F. X. Am. J. Enol. Vitic. 1994, 45(2), 192-200.

24. Moutounet, M.; Masson, G.; Scalbert, A.; Baumes, R.; Lepoutre, J. P.; Puech, J. L. Rev. Fr. Oenol. 1995, 151, 25-31.

25. Conner, J. M.; Paterson, A.; Piggott, J. R. J. Sci. Food Agric. 1993, 62, 169-174.

26. Clyne, J.; Conner, J. M.; Paterson, A.; Piggott, J. R. Int. J. Food Sci. Techn. 1993, 28, 69-81.

27. Piggott, J. R.; Conner, J. M.; Paterson, A.; Clyne, J. Int. J. Food Sci. Technol. 1993, 28, 303-318.

28. Conner, J. M.; Paterson, A.; Piggott, J. R. J. Sci. Food Agric. 1992, 60, 349-353. 
Original articles 